\title{
AUDITORY DISPLAYS OF ELECTRIC POWER GRIDS
}

\author{
Patrick Cowden, Luke Dosiek \\ Union College \\ Department of Electrical, Computer, \\ and Biomedical Engineering \\ Schenectady, NY 12308 \\ patrickjcowden@gmail.com \\ dosiekl@union.edu
}

\begin{abstract}
This paper presents auditory displays of power grid voltage. Due to the constantly changing energy demands experienced by a power system, the voltage varies slightly about nominal, e.g., $120 \pm 2 \mathrm{~V}$ at $60 \pm 0.04 \mathrm{~Hz}$. These variations are small enough that any audible effects, such as transformer hum, appear to have constant volume and pitch. Here, an audification technique is derived that amplifies the voltage variations and shifts the nominal frequency from $60 \mathrm{~Hz}$ to a common musical note. Sonification techniques are presented that map the voltage magnitude and frequency to MIDI velocity and pitch, and create a sampler trigger from frequency deviation. Several examples, including audio samples, are given under a variety of power system conditions. These results culminate in a multi-instrument track generated from the sonification of time-synchronized geographically widespread power grid measurements. In addition, an inexpensive Arduino-based device is detailed that allows for real-time sonification of wall outlet voltage.
\end{abstract}

\section{INTRODUCTION}

AC electric power grids are highly regulated such that under ambient operating conditions the magnitude and frequency of the voltages throughout the system remain virtually unchanged from their nominal values, e.g., $120 \mathrm{~V}$ and $60 \mathrm{~Hz}$ at a residential wall outlet in the United States. If one were to measure the voltage, however, one would notice that in reality there are small variations about nominal as seen in Fig. 1, which displays the residential mains-level voltage magnitude and frequency of the US Eastern Interconnect power system over a period of 1 hour.

This variability about nominal is due to the aggregate effect of small and seemingly random changes in electrical load caused by events such as lights being switched on and off. Indeed, the random component in the measured system outputs is well-modeled as white noise that has been filtered by the power grid system dynamics $[1,2]$. Due to the fact that system dynamics information is embedded in these measured ambient data, they may be analyzed as predictors of overall power grid health and stability, e.g., [2-6]. The data may also be used to detect transient events

\section{(c) (1) ()} Commercial 4.0 International License. The full terms of the License are available at http://creativecommons.org/licenses/by-nc/4.0
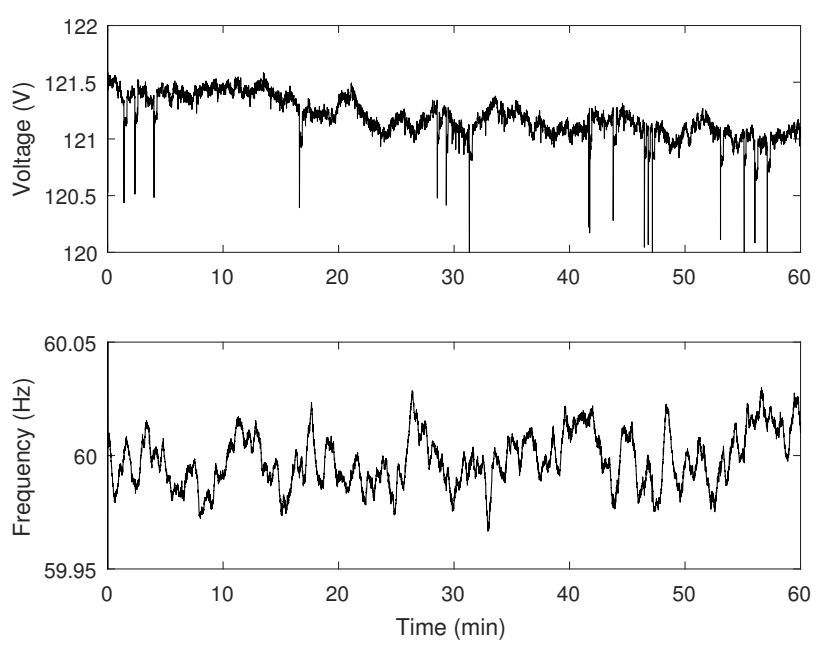

Figure 1: Example voltage magnitude and frequency measured from the United States Eastern Interconnect power system.

such as ringdown-type responses to system disturbances or rogue oscillations due to, e.g., damaged controllers $[7,8]$.

Despite the information-rich signals available in the measured power system outputs, the grid is so tightly controlled that the AC waveforms appear to have constant amplitude and frequency when viewed by the naked eye. Similarly, the audible effects of the AC signals appear to have constant parameters, e.g., the transformer hum that is often heard near substations. With some signal processing, however, one can amplify the ultra low-level variations in the AC waveform so that they are perceivable to the human ear.

This would allow for the use of audio displays to augment existing wide-area situational awareness tools currently used by power grid operators. These include the numerical methods discussed in the aforementioned references, as well as simple visual displays of system parameters such as the spectra of the frequency variations. Indeed, it has been shown in, e.g., [9] that the human ear can be more successful than the eye in perceiving certain spectral features of a signal. Additionally, there is evidence that auditory displays may be used in situational awareness applications; the authors of [10] utilize the sonification of network traffic to detect anomalies such as attempted attacks by hackers.

To the best of the authors' knowledge, the only prior work 
on generating auditory displays for power systems has been conducted by the authors of [11-14]. They created a sonification tool for converting a measure of the energy consumed by household appliances into sounds, e.g., bird songs, that could be incorporated into the ambient soundscape of a person's home life. One of the goals of that project was to increase the awareness of the general public of the characteristics of their home energy use.

This paper focuses on the development of auditory display techniques for power system AC voltage measurements. These signals include a random component, and can be obtained from the multichannel geographically-widespread time-synchronized measurements typically used for wide-area situational awareness by power grid operators. Previous work outside of the power systems realm has demonstrated the feasibility of such a scheme. For example, the authors of $[15,16]$ developed methods of audification and musification, respectfully, of multichannel geographicallywidespread seismograph data. The author of [17] describes the use of random processes, not unlike the random variations in power system voltage, in the composition of Achorripsis, a musical piece created by Iannis Xenakis in 1957 . The auditory display techniques explored in this paper are applied to both simulated and measured voltage data that include both ambient and oscillatory disturbance conditions. The resulting audio waveforms range from the basic audification of a single AC voltage signal to a multiinstrument MIDI-based musification.

This paper is organized as follows. In Section 2, an overview of power system small-signal dynamics is given that includes measurement and preprocessing techniques. Sections 3 and 4 detail the audification and sonification techniques used, and Sections 5 and 6 provide audification and sonification results, including audio samples. Section 7 describes a device that was developed for the realtime sonification of mains voltage, and finally Section 8 provides concluding remarks and a discussion of ongoing and future work.

\section{POWER SYSTEM DYNAMICS AND MEASUREMENTS}

The power system dynamics under consideration here are of extremely low frequency. As seen in Fig. 1, the signals are dominated by a very slow moving process that is due to the speed governor located on each synchronous generator connected to the grid. Supported by the total inertia of turbines and generators, these dynamics are in the sub- $0.1 \mathrm{~Hz}$ range and have large time constants typical of massive mechanical systems. The speed governors work to keep the grid in synchronization, meaning that for a given window in time, the sub- $0.1 \mathrm{~Hz}$ variations in system frequency will be nearly identical throughout the system. The voltage magnitude is similarly controlled, but is in general altered by power transmission and distribution components according to linear circuit theory.

Electromechanical dynamics, ranging from 0.1 to $2.0 \mathrm{~Hz}$, cause signal fluctuations that do vary from location to location. They are caused by turbine dynamics interacting with transmission line dynamics and manifest as groups of generators with speeds that swing with or against other groups of generators. They have low energy compared to the speed governor dynamics, seen in Fig. 1 as the small noisy process superimposed on the low-frequency trend.

Finally, note in Fig. 1 the occasional brief drops in voltage magnitude that are not present in the frequency signal. These are caused by the inrush currents of motors starting up near the measurement device. These brief spikes in current cause a corresponding drop in the voltage magnitude on all lines connected to the same circuit breaker due to the resistive losses in the distribution wires. They do not affect the frequency since they cannot change the rotational inertia of the synchronized generators on the entire power grid. In order to observe similar transients in frequency, a large system-level event is required such as a transmission line failure.

In order to understand how one measures the instantaneous voltage magnitude and frequency discussed so far, one must examine the mathematical expression for the instantaneous voltage:

$$
v(t)=\sqrt{2} V(t) \cos \left(2 \pi f_{o} t+\theta(t)\right)
$$

where $V(t)$ is the root mean squared (RMS) magnitude of the voltage, $f_{o}$ is the nominal system frequency, and $\theta(t)$ is the instantaneous phase angle, which may be expressed as

$$
\theta(t)=\theta_{o}+2 \pi{ }_{0}^{t} \Delta f(\tau) d \tau
$$

where $\theta_{o}$ is the initial phase angle of the sinusoid and $\Delta f(t)$ is the deviation in frequency from nominal such that the instantaneous frequency $f(t)$ is

$$
f(t)=\frac{1}{2 \pi} \frac{d}{d t}\left(2 \pi f_{o} t+\theta(t)\right)=f_{o}+\Delta f(t)
$$

In phasor notation, the instantaneous voltage is a two-element complex number comprised of the magnitude and phase

$$
\bar{V}(t)=V(t) e^{j \theta(t)}=V(t) \measuredangle \theta(t)
$$

where $j$ is the imaginary number. Note that in Fig. $1, V(t)$ is shown in the upper plot, while $f(t)$ is in the lower.

Estimation of the voltage parameters begins with the sampling of the raw voltage waveform followed by a filtering operation that attenuates harmonics, measurement noise, or other interference, allowing the acquired signal to closely resemble (1). Then any one of several signal processing approaches may be taken to obtain $V(t)$ and $f(t)$. They range from the simple, e.g., finding $V(t)$ from a direct calculation of the root-mean-squared value of a period of $v(t)$ and finding $f(t)$ through zero-crossing estimation; to the complex, e.g., FM demodulators, recursive maximum likelihood estimators, etc. See [18] for several examples.

Alternatively, phasor measurement units (PMU) are commercial devices that provide signals known as synchrophasors (named due to their GPS-time synchronized nature) that are estimates of the phasor quantity $\bar{V}(t)$ in (4). The voltage magnitude $V(t)$ is readily available as the synchrophasor magnitude, and $f(t)$ may be found by adding nominal system frequency $f_{o}$ to the numerical derivative of the synchrophasor angle $\theta(t)$, which is $\Delta f(t)$. Note that in this paper, synchrophasor data was used with the audification and sonification techniques outlined in the following two sections.

\section{AUDIFICATION METHOD}

Because the instantaneous power system voltage is already a sinusoid, a simple audification process may be implemented similar to what was developed for seismograph data in [16]. Input to the audification algorithm are the voltage magnitude $V(t)$ and frequency $f(t)$. They are each normalized as

$$
V_{n}(t)=\frac{V(t)-\min (V(t))}{\max (V(t))-\min (V(t))} \in[0,1]
$$




$$
f_{n}(t)=2 \frac{f(t)-\min (f(t))}{\max (f(t))-\min (f(t))}-1 \in[-1,1]
$$

Noting that the expression in (3) implies that the normalized frequency is equivalent to the normalized frequency deviation, the normalized instantaneous phase $\theta_{n}(t)$ is found by numerically integrating $\Delta f_{n}(t)=f_{n}(t)$ and setting initial phase $\theta_{o}=0$. Note this normalization is conducted with reference to the $\mathrm{min} / \mathrm{max}$ values of the particular measurements under investigation such that maximum values of $V(t)$ and $f(t)$ get mapped to 1 and the minimum values get mapped to 0 and -1 , respectively. Other normalization algorithms could be used here, such as normalizing with respect to "typical" $\mathrm{min} / \mathrm{max}$ values under normal power system operating conditions. The exploration of these alternatives is a subject of ongoing work.

To create the audio signal, $V_{n}(t)$ and $\theta_{n}(t)$ are upsampled using linear interpolation to $44.1 \mathrm{kHz}$ from, e.g., the $30 \mathrm{~Hz}$ reporting rate of a PMU. Then, audio signal $a(t)$ is formed as

$$
a(t)=V_{n}(t) \cos \left(2 \pi f_{a}+\alpha \theta_{n}(t)\right)
$$

with user-tunable parameters $f_{a}$, which defines the "middle" note (e.g., $261.63 \mathrm{~Hz}$ for middle $\mathrm{C}$ ), and $\alpha$, which determines maximum excursion in pitch from $f_{a}$.

Once constructed, $a(t)$ provides a simple auditory display for the nonstationarity of practical power grid voltage.

\section{SONIFICATION METHODS}

Three different sonification techniques were implemented using the parameter mapping techniques from [19]. The first involves mapping the voltage magnitude $V(t)$ and frequency $f(t)$ to MIDI note velocity and pitch, respectively. The second technique consists of using a threshold detector on frequency fluctuation $\Delta f(t)$ to create a MIDI-based sampler trigger. Lastly, the third technique derives the MIDI note length from the sampling rate of $V(t)$ and $f(t)$. The data processing for each of the sonification algorithms is implemented in Python, and MIDI files are exported using the MIDI-Util Python library [20].

\subsection{MIDI Note Velocity and Pitch Mapping}

For this method, samples of voltage magnitude $V(t)$ are converted to an array of MIDI velocities and samples of voltage frequency deviation $f(t)$ are converted to an array of MIDI pitches. To create the MIDI velocity values, the normalized voltage magnitude used in the audification process, $V_{n}(t)$, is scaled by 127 to span between 0 and 127 (since there are 128 possible MIDI velocity values) and rounded to the nearest integer.

Creating MIDI pitch values is slightly more complicated as the user may specify both the musical key and the number of octaves above/below the middle note that are to be spanned by the mapping of $\Delta f_{n}(t)$. First, the range $[-1,1]$ is divided into $N$ equally sized bins corresponding to the total number of notes included in the desired number of octaves. Then, the user-selected key is used to determine which notes (and MIDI pitch values) correspond to each bin. Note that if the user specifies an invalid key value, the algorithm defaults to $\mathrm{C}$ major. Finally, each value of $\Delta f_{n}(t)$ is assigned a MIDI pitch based on which bin it belongs to.

For example, if one desired sonification in the key of $\mathrm{C}$ major with 1 octave above/below C4, then 2 octaves with 7 notes each plus the middle $\mathrm{C}$ note would require 15 bins in the range $[-1,1]$ as seen in Table 1 . So if three consecutive samples of
Table 1: Example MIDI Pitch Mapping in C Major with 1 octave above and below middle.

\begin{tabular}{c|c|c|c}
\hline \hline Bin & $\Delta f_{n}$ Range & Note & MIDI Pitch \\
\hline 1 & {$[-1,-0.8667)$} & C3 & 48 \\
2 & {$[-0.8667,-0.7333)$} & D3 & 50 \\
$\vdots$ & $\vdots$ & $\vdots$ & $\vdots$ \\
8 & {$[-0.0667,0.0667)$} & C4 & 60 \\
$\vdots$ & $\vdots$ & $\vdots$ & $\vdots$ \\
14 & {$[0.7333,0.8667)$} & B5 & 71 \\
15 & {$[0.8667,1]$} & C5 & 72 \\
\hline \hline
\end{tabular}

$\Delta f_{n}(t)$ were $[-0.91,-0.99,0.81]$, then the MIDI pitches would be $[48,48,71]$.

\subsection{Sampler Triggering}

This method analyzes the absolute value of the normalized voltage frequency deviation $\Delta f_{n}(t)$ to create an array of MIDI velocity values that can be mapped to sampler triggering. A threshold $\gamma \in$ $[0,1]$ is applied such that the MIDI velocity $M_{v}$ is

$$
M_{v}(t)=\begin{array}{ll}
0 & \text { if }\left|\Delta f_{n}(t)\right| \leq \gamma \\
127 & \text { if }\left|\Delta f_{n}(t)\right|>\gamma
\end{array}
$$

To finalize the sampler triggering, the MIDI pitch is fixed at 60 , the value used by MIDI samplers to play back the original audio sample. This may then be opened with an appropriate sampling device which will play a particular sound, e.g., a snare drum hit, each time the power system frequency $f(t)$ deviates far enough from nominal.

\subsection{MIDI Note Length Assignment}

If real-time sonification is not necessary, one may simply assign a constant note length, e.g. an eighth note, to all data samples. Or, one may randomly assign standard note lengths such as sixteenth, eighth, or quarter notes. Depending on the tempo of the musical piece and the sampling rate of the voltage measurement, this will in general result in an audio recording that is of a different duration than the power system voltage recording. For example, eighth notes at $120 \mathrm{bpm}$ occupy 0.25 seconds each. If constant eighth note lengths are applied to 10 seconds of voltage data recorded at 60 samples per second, the resultant audio file will be comprised of 600 eighth notes and last 150 seconds.

When real-time sonification is desired, the MIDI note length may be set in decimal quarter note format based on the sampling rate of the streaming voltage measurements. Given sampling rate $f_{s}$ in samples per second and musical tempo $T$ in beats per minute, the MIDI note length $M_{l}$ in beats per sample is found as

$$
M_{l}=\frac{T}{60 f_{s}}
$$

For the above example, this would result in an $M_{l}$ of 0.0333 beats per sample. When compared to using constant eighth notes, which would correspond to 0.5 beats per sample, the notes would be 
played at a much higher speed, but the audio and voltage signals would have identical time durations, thus enabling real-time operation.

\section{AUDIFICATION RESULTS}

This section presents the results of the previously described audification process applied to both measured and simulated power grid voltage under ambient and oscillatory conditions.

\subsection{Ambient Conditions}

Thirty seconds of voltage magnitude and frequency were measured from a wall outlet at Union College in Schenectady, NY, as seen in Fig. 2, where one of the aforementioned motor startup effects can be seen in the voltage magnitude. The audification process described in Section 3 was applied with $f_{a}=261.63 \mathrm{~Hz}$ and $\alpha=$ 75 . In the resulting audio track, which can be found at

https://soundcloud.com/pcowden/ambientdata-audification

the fluctuations of voltage magnitude can be heard as changes in volume, while the frequency fluctuations can be heard as the slowly changing pitch of the tone.

\subsection{Oscillatory Conditions}

One minute of voltage magnitude and frequency was simulated in MATLAB using the miniWECC, a reduced-order linearized model of the Western Electricity Coordinating Council (WECC) power system [21]. A forced oscillation scenario was created by injecting a $0.35 \mathrm{~Hz}$ square wave as real power on a particular generator at the 20-second mark. Forced oscillations are deterministic periodic signals that appear in otherwise ambient data due to a periodic system input such as a poorly tuned controller, broken equipment, or a cyclic load. See [22] for a detailed discussion of power system forced oscillations. The simulated data is shown in Fig. 3.

The audification process was applied with $f_{a}=261.63 \mathrm{~Hz}$ and $\alpha=175$, resulting in the audio file found at https://soundcloud.com/pcowden/forcedoscillation.

The power system forced oscillation is clearly heard as periodic fluctuations in volume and pitch.

\section{SONIFICATION RESULTS}

This section presents the results of the previously described sonification process applied to measured power grid voltage under ambient and transient conditions. It also includes a multi-instrument composition derived from multiple voltage measurements. Note that the reFX Nexus VST synthesizer plugin was used in each of the examples below.

\subsection{Ambient Conditions}

Sonification was performed on the ambient data from Fig. 2. MIDI note velocity and pitch mapping were used in the key of $\mathrm{Bb}$ major with 2 octaves above and below the middle $\mathrm{Bb}$ note. The sampling rate of the voltage data was 4 samples per second, and the tempo of the audio was $120 \mathrm{bpm}$. The MIDI note lengths were first set to be all eighth notes, and then were chosen randomly among quarter, eighth, and sixteenth notes. The MIDI file was played by the
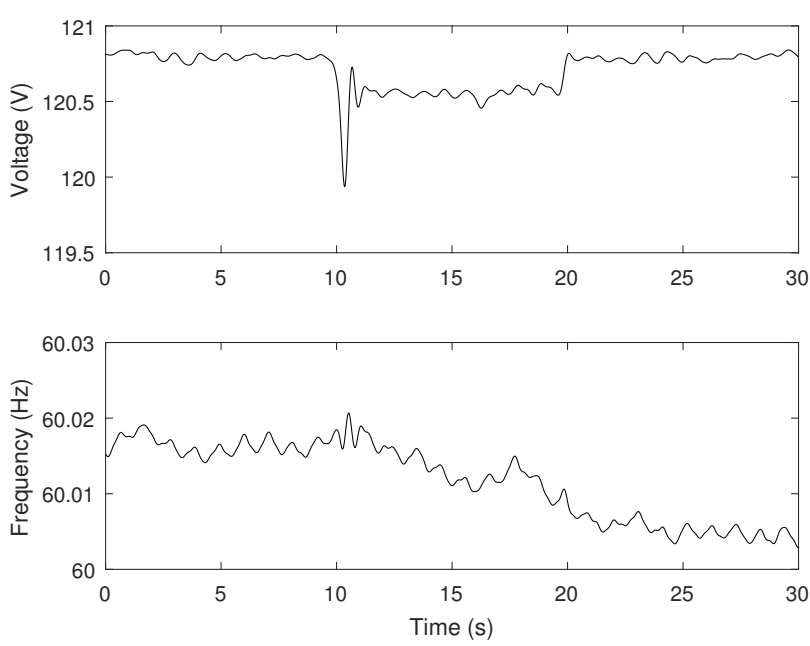

Figure 2: Ambient voltage data measured from wall outlet at Union College in Schenectady, NY.
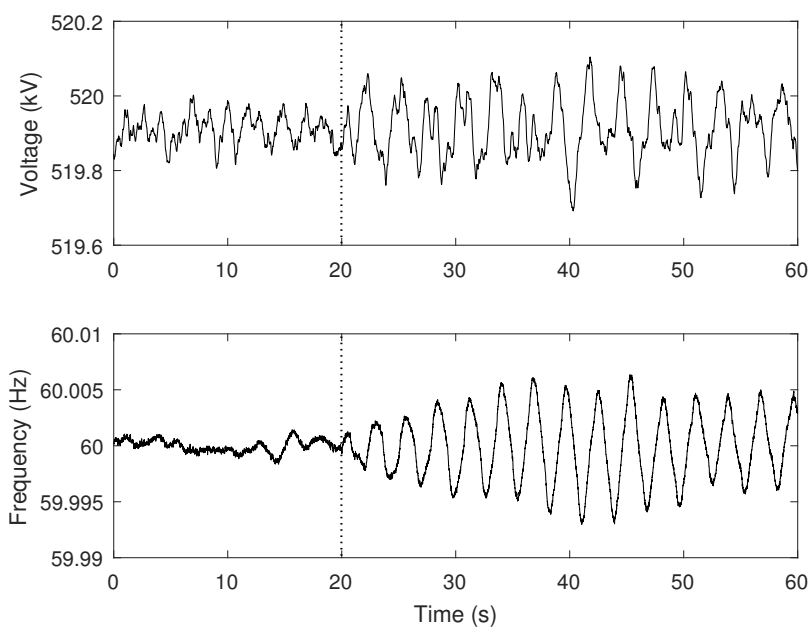

Figure 3: Ambient voltage data simulated from the miniWECC system with $0.35 \mathrm{~Hz}$ forced oscillation starting at 20 seconds.

synthesizer using the "UK Pikes 1 Pluck" synth, creating the following audio pieces:

Constant note length:

https://soundcloud.com/pcowden/power-gridambient-data-sonification

Random note length:

https: / / soundcloud. com/pcowden/powergrid-ambient-data-sonification-bb-majorrandomized-note-length

In the resulting tracks, the fluctuations of voltage magnitude can be heard as changes in note volumes while fluctuations of the voltage frequency can be heard as varying note pitches. While both tracks provided an auditory display of the power system voltage, the version with the random note lengths provided a more melodic experience. 

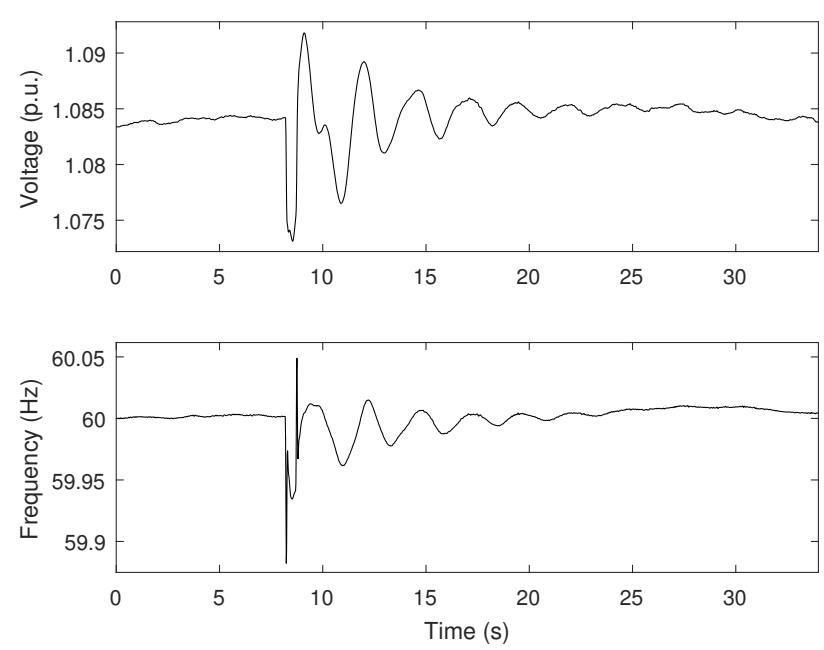

Figure 4: Voltage data measured from the WECC system during a transient ringdown event.

\subsection{Transient Ringdown Response}

A stable power system responds to a large system disturbance through a damped natural oscillation process referred to in the power community as a "ringdown response." The frequencies and dampings of the ringdown are defined by the natural oscillatory modes of the power system. If the system disturbance is large enough, the ringdown signal will dominate, providing an extremely high SNR signal that engineers may use to estimate the system modes with great accuracy.

An example of one such ringdown response that was measured in the WECC system is given in Fig. 4. The voltage magnitude is given in per-unit (p.u.) in order to mask the location of the measurement due to confidentiality agreements.

For the sonification of this data, the MIDI velocity and pitch were mapped from the voltage data in the key of $\mathrm{F}$ major spanning 4 octaves above/below the middle note. As seen in Fig. 5, the synthesizer display of the MIDI values closely resemble the power grid measurements of Fig. 4. To simulate a real-time environment, the MIDI note lengths were set using (9) with a tempo of $150 \mathrm{bpm}$ and a power grid data sampling rate of 10 samples per second, resulting in a MIDI note length of 0.25 in decimal quarter notes, which represents a sixteenth note. The resultant MIDI file was processed by the synthesizer using the "Waterhouse Pikes Pluck" synth to create the following audio file:

https://soundcloud.com/pcowden/transientring-down-response-real-time-f-major

\subsection{Multichannel Ambient Data (Musification)}

Time-aligned synchrophasor data from six geographically widespread locations in the WECC power grid were sonified to create a multi-instrument composition. The voltage magnitude (in per-unit) and the frequency are shown in Fig. 6. The locations of measurements are withheld and the voltage magnitude shown in the accompanying figure was normalized before converting to per-unit in order to honor confidentiality agreements.

The sampling rate for the synchrophasors was 60 samples per second, and 640 samples from each measurement location were

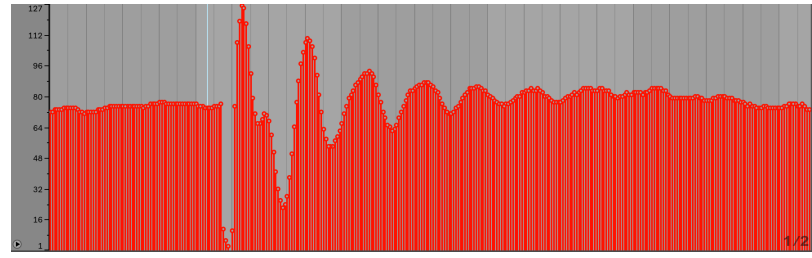

(a) MIDI note velocity

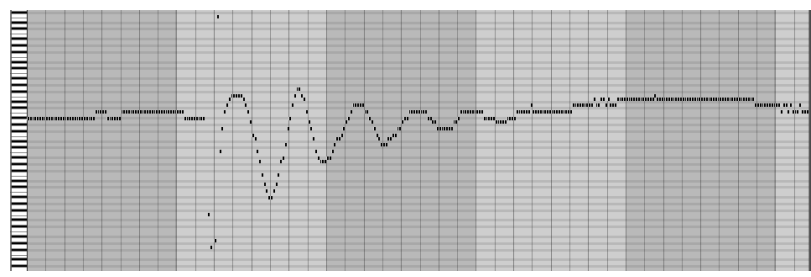

(b) MIDI note pitch

Figure 5: Synthesizer display of MIDI note velocity and pitch mapped from the voltage magnitude and frequency of Fig 4.
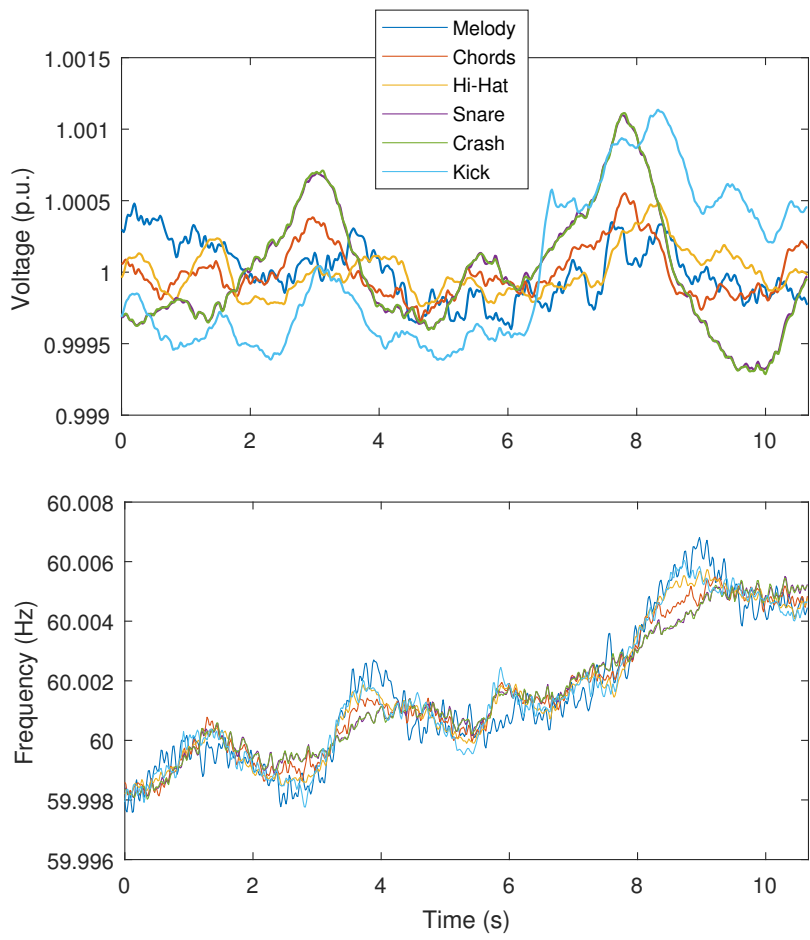

Figure 6: Voltage data measured from the WECC system during ambient conditions used in the multichannel sonification example.

analyzed, resulting in 10.667 seconds of power grid data. The musical piece was created at $120 \mathrm{bpm}$ and one sampling period of the voltage data was assigned to be a sixteenth note, or 0.125 seconds, resulting in an 80-second musical composition.

The first measurement location was sonified with velocity and pitch mapping to provide a lead melody in the key of A major spanning 2 octaves above/below the middle note. The MIDI note lengths were randomly chosen as either $1,2,3$, or 4 sixteenth notes. In order to preserve the time-aligned nature of the syn- 
chrophasor data, if a note longer than 1 sixteenth note was chosen, the sonification algorithm would skip over the appropriate number of samples. For example, if the first note length was 3 sixteenth notes, then the first sample of voltage data would be sonified, and the next two samples would be skipped. The "Waterhouse Pikes Pluck" synth was applied to the resultant MIDI data.

The next measurement location was chosen to play chords with the same key and octave span as the melody. Every $16^{\text {th }}$ sample of the voltage data was sonified, beginning with the $1^{\text {st }}$ sample, and assigned a MIDI note length corresponding to a whole note. The "Andromeda Pad" chord synth was applied to the MIDI data.

The third measurement location was sonified using sampler triggering with a threshold of 0.15 to trigger a hi-hat sample. In order to create a rhythm common to dance music, where the hi-hat is only heard on even eighth notes (the "and" of each beat), every $4^{\text {th }}$ sample of voltage data was sonified, beginning with the $3^{\text {rd }}$ sample, and assigned a MIDI note length of a quarter note.

The fourth measurement location was sonified using sampler triggering with a threshold of 0.1 to trigger a snare drum sample. In order to create the common rhythm of snare drums only being hit on the back beats, every $8^{t h}$ sample of voltage data was sonified, beginning with the $5^{t h}$ sample, and assigned a half note MIDI note length.

The fifth measurement location was sonified using sampler triggering with a threshold of 0.275 to trigger a crash cymbal sample. Every $8^{t h}$ sample of voltage data was sonified, beginning with the $7^{t h}$ sample, and assigned a half note MIDI note length. This placed the cymbal crashes on the eighth note (the "and") after a snare hit.

The last measurement location was sonified using sampler triggering with a threshold of 0.02 to trigger a kick drum sample. Every $4^{t h}$ sample was sonified, beginning with the $1^{\text {st }}$ sample, and assigned a MIDI note length corresponding to a quarter note. This timing coupled with the low trigger threshold created a nearly constant quarter note kick drum feel typical to this genre of music.

The composition, which can be heard at

https: //soundcloud.com/pcowden/multi-

channel-power-grid-musification-pure

reflects the synchronized nature of AC power grid dynamics in an auditory display that is truly musical in nature. In this track, every sound has been controlled by power grid data.

Transitional audio samples were manually added and the melody and chord tracks were processed with a kick drum sidechain compression effect to create the track that can be heard at https://soundcloud.com/pcowden/multichannel-power-grid-musification-edited

This provides an example of how the sonified power grid data could be incorporated into the regular work flow of an artist. The resulting composition is a piece of music that contains elements under both human and power grid control.

\section{REAL-TIME SONIFICATION DEVICE}

A sonification device was designed that allows for the conversion of wall-outlet AC voltage to a MIDI signal in real time. As seen in Fig. 7, the device consists of five main components. The first is a center-tapped 5:1 transformer that takes the 120-VAC voltage from the wall socket connection and steps it down to a safe level. The center tap is connected to the wall socket earth ground, providing three output voltages: 24-VAC line-to-line, and a pair of 12-VAC line-to-ground voltages that are anti-phase with each other.

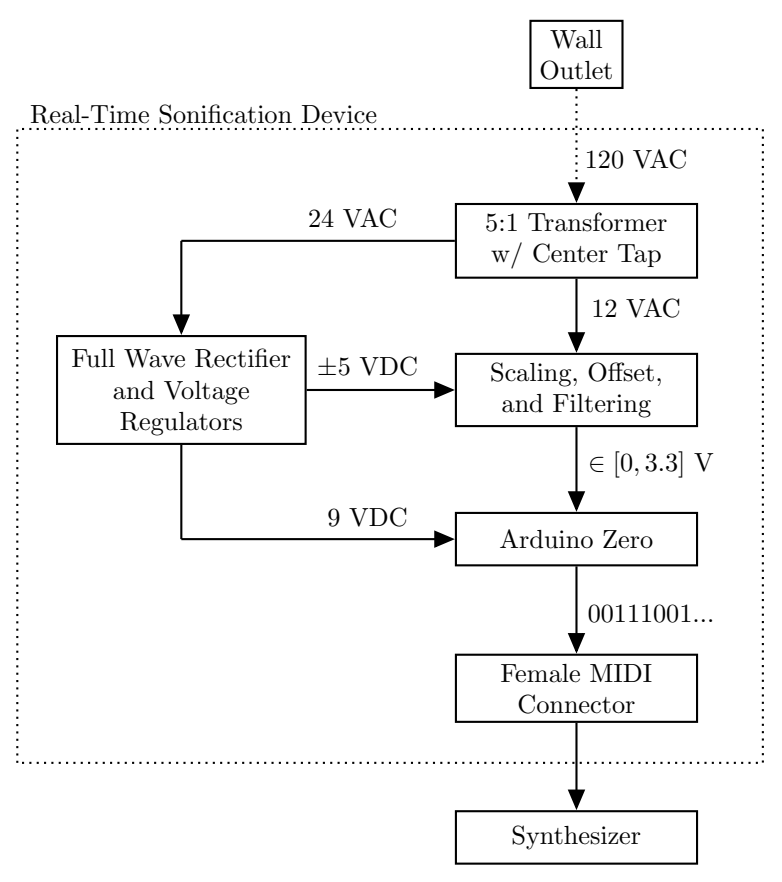

Figure 7: Flow chart of real time sonification device.

One of the 12-VAC transformer outputs is connected to the second component of the device which scales, offsets, and filters the voltage for input to the Arduino. First, a voltage divider is used to reduce the signal to approximately 1 VAC. Then an opamp circuit is used to add approximately 2 VDC to the signal. Finally, the signal is passed through a first-order RC low-pass filter with cutoff of approximately $61 \mathrm{~Hz}$ to remove harmonics and high frequency noise. All components are referenced to the transformer center tap. The result is a clean sinusoid with DC offset such that it satisfies the required 0 to $3.3-\mathrm{V}$ range of the Arduino analog input pin.

The Arduino microcontroller serves as the main component of the sonification system. An Arduino Zero was used for its high precision and accuracy with regards to analog-to-digital conversion and subsequent signal processing. It completes all of the signal measurement, processing, parameter-mapping sonification, and MIDI signal creation via software.

First, it estimates voltage magnitude and frequency from the sampled analog voltage input. The software operates on windows of six periods of $60-\mathrm{Hz}$. For each data window, the mean is subtracted to remove the DC offset added by the op-amp circuit. Then the voltage magnitude is found by calculating the root mean square (RMS) of the zero-mean signal. The frequency is estimated through a zero-crossing detection technique. The signal is scanned for changes in sign, and the actual zero crossing time $t_{z c}$ is estimated through linear interpolation

$$
t_{z c}=t_{1}-v_{1} \frac{t_{2}-t_{1}}{v_{2}-v_{1}}
$$

where $t_{1}$ and $v_{1}$ refer to the time and voltage values of the sample just before a change in sign, and $t_{2}$ and $v_{2}$ refer to the values just after the sign change. Given that there are six cycles of $60-\mathrm{Hz}$ 
voltage per analysis window, there will be twelve zero crossings, collected in vector $\underline{t}_{z c}$. The frequency estimate for the window is derived from an averaging process that is robust to timing errors and small sampling delays. It first averages frequencies estimated from odd- and even-numbered zero crossings separately, then takes the mean of those averages as the final frequency estimate:

$$
\begin{aligned}
\hat{f}_{1} & =\frac{1}{5} \sum_{i=1}^{5} \frac{1}{\underline{t}_{z c}[2 i+1]-\underline{t}_{z c}[2 i-1]} \\
\hat{f}_{2} & =\frac{1}{5} \sum_{i=1}^{5} \frac{1}{\underline{t}_{z c}[2 i+2]-\underline{t}_{z c}[2 i]} \\
\hat{f} & =\frac{1}{2} \quad \hat{f}_{1}+\hat{f}_{2}
\end{aligned}
$$

where $\underline{t}_{z c}[i]$ is the $i^{t h}$ element of $\underline{t}_{z c}$.

With voltage magnitude and frequency estimated, the previously discussed sonification techniques are implemented. Prior to uploading the software to the Arduino, the user selects the sonification parameters, i.e., musical key, number of octaves, triggering threshold, tempo, and note length technique. The software implements the sonification and converts to a binary MIDI signal that is sent to a digital output pin.

The MIDI notes created by the software are passed through one of the Arduino digital output pins to a female MIDI connector following the MIDI OUT electrical specifications for 3.3-V signaling [23]. Any synthesizer may then connect to the device via the female MIDI connector to initiate musical playback.

Note that both the Arduino and the op-amp require DC power supplies. These were created by the fifth and final component of the device, which first converts the 24-VAC transformer output to a DC signal through a full-wave bridge rectifier with smoothing capacitors. This DC signal is then fed to voltage regulators that provide the \pm 5 -VDC required by the op-amp and the 9 -VDC required by the Arduino. Once again, all components were referenced to the transformer center tap.

Whether a student in the classroom setting, a musician looking to incorporate the environment into their compositions, or a casual observer, it is intended that the development of the Arduino-based sonification device will provide a simple and inexpensive way of creating auditory displays of the power grid voltage nearest them.

\section{CONCLUSIONS}

The methods developed for this research successfully generated interesting auditory representations of power grid data. The audification and sonification algorithms are easy to use and can be applied to measured power grid voltage from virtually anywhere. For those that are unaware that the power grid voltage parameters are not always $120-\mathrm{V}$ at $60-\mathrm{Hz}$, these auditory displays allow for a simple comprehension of what is really happening in a power system. Furthermore, since the fluctuations in voltage are due to the physical properties of the power grid responding to the sum of society's ever-changing energy demands, these auditory displays allow one to experience the very heartbeat of an incredibly complex system that is only ever given a second thought when the power goes out.

Ranging from the other-worldly theramin-sounding audification tracks, to the synthesizer-based sonification pieces, it has been shown that in addition to being informative these auditory displays are rather entertaining. The multi-channel musification composition sounds like a dance track that was entirely human-produced, when in fact the power grid was playing the instruments. It is an entertaining composition that speaks to the significance of auditory displays, and shows how the intersection of music and power engineering can be used to spark interest in both areas.

An important area of future work is the consideration of these approaches as a tool for actual power system operators to monitor the overall health of the system, or to detect events such as forced oscillations or ringdown responses. Currently this is achieved with visual indicators, e.g., dials, graphs, or raw numbers, that are the results of traditional numerical analysis. A study with grid operators should be conducted in which they are presented with auditory displays of typical grid parameters in order to determine practical usefulness.

Another area of future work is the exploration of how the various audification and sonification algorithm parameters should be selected. For the results in this paper, they were tuned until the authors found an audio track that was pleasing. Consultation with music theorists and surveys with listeners should be conducted to determine which settings make for a more globally pleasing sound.

Lastly, it must be noted that the multi-channel musification tracks are rather prototypical in nature. The six power grid measurement locations chosen for sonification were done so based solely on the availability of the data, and they were randomly assigned to instruments. This created a false hierarchy of importance among the power system measurement channels since the listener will tend to assign greater importance to the melody track as compared to, e.g., the hi-hat. Furthermore, the particular instruments used in the synthesizer were chosen simply based on the musical tastes of the authors, and did not relate in any physical way to the nature of the power grid.

The next step in improving upon the power grid musification process is to address these issues. Power system measurement location may be ordered by importance based upon grid topology. For example, measurements made closest to major generation stations or load centers could be assigned higher "importance" than those obtained from the middle of a long transmission corridor if one associates importance with energy sources and sinks. Or, one could assign "importance" of a measurement location based upon its level criticality to the overall reliability of the power grid.

One could also explore the fact that synchrophasor measurements typically include current phasors in addition to the voltage phasors, implying that one can easily calculate both real and reactive power flow quantities. Thus, a scheme could be developed that assigns instrumentation based on signal type. For example, since the voltage magnitude and frequency are the quantities that are most tightly controlled by the grid, they could be used to provide the rhythm section, while the more wildly varying current and/or power values could control the melodic instruments.

Finally, a study into integrating properties of the power grid into the selection of the instruments used in the synthesizer should be conducted. Perhaps there is a musical genre better suited to represent power grid dynamics than the electronic dance music presented here. The use of audio samples such as the bird songs used in [11-14] should also be considered.

\section{ACKNOWLEDGMENT}

The authors would like to thank Professors Palmyra Catravas and Nick Webb at Union College and Dr. Ryan McGee of Life Orange 
LLC for their insightful discussions throughout this project.

\section{REFERENCES}

[1] P. Kundur, N. J. Balu, and M. G. Lauby, Power system stability and control. McGraw-hill New York, 1994, vol. 7.

[2] J. W. Pierre, D. J. Trudnowski, and M. K. Donnelly, "Initial results in electromechanical mode identification from ambient data," IEEE Transactions on Power Systems, vol. 12, no. 3, pp. 1245-1251, 1997.

[3] D. J. Trudnowski, J. W. Pierre, N. Zhou, J. F. Hauer, and M. Parashar, "Performance of three mode-meter blockprocessing algorithms for automated dynamic stability assessment," IEEE Transactions on Power Systems, vol. 23, no. 2, pp. 680-690, 2008.

[4] N. Zhou, D. J. Trudnowski, J. W. Pierre, and W. A. Mittelstadt, "Electromechanical mode online estimation using regularized robust RLS methods," IEEE Transactions on Power Systems, vol. 23, no. 4, pp. 1670-1680, 2008.

[5] L. Dosiek, J. W. Pierre, and J. Follum, "A recursive maximum likelihood estimator for the online estimation of electromechanical modes with error bounds," IEEE Transactions on Power Systems, vol. 28, no. 1, pp. 441-451, 2013.

[6] S. N. Sarmadi and V. Venkatasubramanian, "Electromechanical mode estimation using recursive adaptive stochastic subspace identification," IEEE Transactions on Power Systems, vol. 29, no. 1, pp. 349-358, 2014.

[7] J. Follum and J. W. Pierre, "Detection of periodic forced oscillations in power systems," IEEE Transactions on Power Systems, vol. 31, no. 3, pp. 2423-2433, 2016.

[8] Y. Song, W. Wang, Z. Zhang, H. Qi, and Y. Liu, "Multiple event detection and recognition for large-scale power systems through cluster-based sparse coding," IEEE Transactions on Power Systems, vol. 32, no. 6, pp. 4199-4210, 2017.

[9] R. L. Alexander, J. A. Gilbert, E. Landi, M. Simoni, T. H. Zurbuchen, and D. A. Roberts, "Audification as a diagnostic tool for exploratory heliospheric data analysis," in 17th International Conference on Auditory Display (ICAD-2011), June 2011.

[10] P. Vickers, C. Laing, M. Debashi, and T. Fairfax, "Sonification aesthetics and listening for network situation awareness," in Conference on Sonification of Health and Environmental Data (SoniHED), September 2014.

[11] D. Lockton, F. Bowden, C. Brass, and R. Gheerawo, "Birdwattching: exploring sonification of home electricity use with birdsong," in Conference on Sonification of Health and Environmental Data (SoniHED), September 2014.

[12] _ _ "Powerchord: Towards ambient appliance-level electricity use feedback through real-time sonification," in 8th International Conference on Ubiquitous Computing \& Ambient Intelligence (UCAmI 2014), December 2014.

[13] D. Lockton, F. Bowden, and C. Matthews, "Powerchord: Exploring ambient audio feedback on energy use," in Living Labs: Design and Assessment of Sustainable Living, D. Keyson, O. Guerra-Santin, and D. Lockton, Eds. Berlin: Springer, 2017, ch. 23, pp. 297-308.
[14] S. Clune and D. Lockton, "Design for behaviour change and sustainability," in Design for Behaviour Change: Theories and practices of designing for change, K. Niedderer, S. Clune, and G. Ludden, Eds. London: Routledge, 2017.

[15] F. Dombois, "Using audification in planetary seismology," in 7th International Conference on Auditory Display (ICAD2001), July 2001.

[16] R. McGee and D. Rogers, "Musification of seismic data," in 22nd International Conference on Auditory Display (ICAD2016), June 2016.

[17] E. Childs, "Achorripsis: a sonification of probability distributions," in 8th International Conference on Auditory Display (ICAD-2002), July 2002.

[18] B. Boashash, "Estimating and interpreting the instantaneous frequency of a signal. ii. algorithms and applications," Proceedings of the IEEE, vol. 80, no. 4, pp. 540-568, 1992.

[19] F. Grond and J. Berger, "Parameter mapping sonification," The sonification handbook, pp. 363-397, 2011.

[20] M. C. Wirt. (2016) MIDIUtil - MIDIUtil 1.1.1 documentation. [Online]. Available: http://midiutil.readthedocs.io/en/ latest/

[21] D. Trudnowski, D. Kosterev, and J. Undrill, "PDCI damping control analysis for the western north american power system," in 2013 IEEE Power Energy Society General Meeting, July 2013, pp. 1-5.

[22] J. D. Follum, F. K. Tuffner, L. A. Dosiek, and J. W. Pierre, "Power system oscillatory behaviors: Sources, characteristics, \& analyses," Pacific Northwest National Lab.(PNNL), Richland, WA (United States), Tech. Rep., 2017.

[23] "MIDI 1.0 electrical specification update," MMA Techincal Standards Board/AMEI MIDI Committee," CA-033, 2014. 